\title{
Solventless Synthesis of Imines Derived from Diphenyldisulphide Diamine or $p$-Vanillin
}

\author{
DANY JEAN -NOËL UPPIAH, MINU GUPTA BHOWON \\ and SABINA JHAUMEER LAULLOO* \\ Department of Chemistry, Faculty of Science, \\ University of Mauritius, Mauritius. \\ sabina@uom.ac.mu
}

Received 10 April 2009; Accepted 5 June 2009

\begin{abstract}
A solventless synthesis can reduce environmental contamination and can be more convenient than solvent-based synthesis. A series of Schiff bases were synthesized in high yield $>90 \%$ under solvent free conditions with negligible waste. All the synthesized products were characterized on the basis of spectral, elemental analysis and melting points and were compared with the data of the products obtained from classical method
\end{abstract}

Keywords: Solvent -free, Imines, Diphenyldisulphide diamine, $p$-Vanillin.

\section{Introduction}

Recently, great efforts have been made on developing chemical technologies that can reduce or eliminate the use of organic solvents in synthesis. Large-scale use of organic solvents has been proved to be environmental hazards ${ }^{1}$. Schiff base compounds are well known because of their wide applications and are useful intermediates in organic synthesis ${ }^{2,3}$. These types of compounds have normally been synthesized by traditional methods using organic solvents. Recently, a few papers reported modern synthetic protocols where benign solvents (water or supercritical $\left.\mathrm{CO}_{2}\right)^{4,5}$, recyclable reactions medium $(\mathrm{PPG})^{6}$ and solvent-less conditions ${ }^{7-10}$ have been used. These conditions are so simple as to be considered almost facile yet generate products in good yields, which give them attractive green chemistry metrics.

Herein, we report a simple and versatile route to mono and bis-imine Schiff bases in good yields. This study investigates the synthesis and characterization of imine products using solvent free conditions and compared with products obtained from traditional methods. 


\section{Experimental}

Melting points were uncorrected and were determined on a Stuart Scientific Electric melting point apparatus. Aldrich and BDH chemicals were used. Furan 2-carboxaldehyde was distilled prior to use. IR spectra $(\mathrm{KBr})$ were recorded on a Mattson 1000 FT IR spectrometer in the range $400-4000 \mathrm{~cm}^{-1} .{ }^{1} \mathrm{H}$ and ${ }^{13} \mathrm{C}$ NMR spectra were recorded on a Bruker spectrometer at $250 \mathrm{MHz}$. Elemental analyses were obtained with a LECO 932 CHNS Mattson 1000 Spectrophotometer. Magnetic susceptibility of the complexes was performed at room temperature in Sherwood scientific magnetic balance.

\section{General experimental procedure}

In a typical solventless experiment the aldehyde ( 2 equivalents) was added to the diphenyldisulphides diamine (1 equivalent) and the mixture was grinded in a mortar and pestle at room temperature for 5 to $20 \mathrm{~min}$ afforded the bis-imine product as a paste or a solid. The paste after leaving for half an hour solidified into a solid. If required, analytically pure product can be obtained by washing with hot ethanol or diethyl ether or recrystallised from ethanol.

For compounds (9-12) for vanillin the solid after grinding was dissolved in minimum amount of ethanol and poured on ice. Analytically pure compound was washed with hot water.

The analyses (mp, IR, NMR, CHNS data) of compounds were consistent with literature values ${ }^{11-13}$.

\section{$N, N^{\prime}$ (1,1'-Dithiobisphenylene) bisbenzyldeimine (1)}

Colour: yellow; ${ }^{1} \mathrm{H}$ NMR $\left(\mathrm{CDCl}_{3}\right): \delta \mathrm{H}$ 8.51(s, 2H), 8.01-7.87(m, 4H), 7.57-7.54(m, 6H), 7.287.22(m, 8H); ${ }^{13} \mathrm{C}$ NMR: $\delta \mathrm{C} 160.1,149.0,136.1,132.1,131.7,129.2,128.8,127.0,126.0$, 118.3; $\mathrm{C}_{26} \mathrm{H}_{20} \mathrm{~N}_{2} \mathrm{~S}_{2}$ found (Calc.) C 73.05(73.5), H 4.2(4.7), N 6.9(6.6), S 15.5(15.1).

\section{$N, N^{\prime}$ (1,1'-Dithiobisphenylene) bis-2-chlorobenzyldeimine (2)}

Colour: yellow; ${ }^{1} \mathrm{H}$ NMR $\left(\mathrm{CDCl}_{3}\right): \delta \mathrm{H} 8.98(\mathrm{~s}, 2 \mathrm{H}), 8.40(\mathrm{~d}, 2 \mathrm{H}), 7.66(\mathrm{~d}, 2 \mathrm{H}), 7.26-6.58(\mathrm{~m}$, $12 \mathrm{H}) ;{ }^{13} \mathrm{C}$ NMR: $\delta \mathrm{C} 156.5,136.2,135.2,132.5,130.7,130.0,129.4,129.2,127.4,127.3$, 127.1, 126.1, 117.5; $\mathrm{C}_{26} \mathrm{H}_{18} \mathrm{C}_{12} \mathrm{~N}_{2} \mathrm{~S}_{2}$ found (Calc.) C 62.8(63.2), H 3.5(3.6), N 5.9(5.7), S 13.5(13.0).

\section{$N, N^{\prime}$ (1,1'-Dithiobisphenylene) bis-2-nitrobenzyldeimine (3)}

Colour: yellow; ${ }^{1} \mathrm{H}$ NMR $\left(\mathrm{CDCl}_{3}\right): \delta \mathrm{H} 8.59(\mathrm{~s}, 2 \mathrm{H}), 8.36(\mathrm{~m}, 4 \mathrm{H}), 8.15(\mathrm{~m}, 4 \mathrm{H}), 7.64(\mathrm{~m}, 2 \mathrm{H})$, 7.26-7.13(m, 6H), ${ }^{13} \mathrm{C}$ NMR: $\delta \mathrm{C} 157.0,149.5,147.8,141.4,132.5,129.7,128.1,127.3$, 127.1, 126.1, 124.1, 117.0; $\mathrm{C}_{26} \mathrm{H}_{18} \mathrm{~N}_{4} \mathrm{O}_{4} \mathrm{~S}_{2}$ found (Calc.) $\mathrm{C}$ 61.3(60.6), $\mathrm{H}$ 3.1(3.5), $\mathrm{N}$ 10.8(10.8), S 12.0(12.4).

$N, N^{\prime}$ (1,1'-Dithiobisphenylene) bisfurfurylimine (4)

Colour: yellow; ${ }^{1} \mathrm{H}$ NMR $\left(\mathrm{CDCl}_{3}\right): \delta \mathrm{H}$ 8.28(s, 2H), 7.68-7.61(m, 4H), 7.15(td, 4H), 7.05(m, 4H), $6.5(\mathrm{~m}, 2 \mathrm{H}) ;{ }^{13} \mathrm{C} \mathrm{NMR}: \delta \mathrm{C} 177.9,152.2,148.9,147.9,146.1,131.9,127.0,126.9,126.0$, 117.2, 116.7, 112.4; $\mathrm{C}_{26} \mathrm{H}_{18} \mathrm{~N}_{4} \mathrm{O}_{4} \mathrm{~S}_{2}$ found (Calc.) C 66.1(65.3), H 3.9(3.9), N 7.4(6.9).

\section{$N$, $N$ ' (1,1'-Dithiobisphenylene) bis-o-anisalimine (5)}

Colour: yellow; ${ }^{1} \mathrm{H}$ NMR $\left(\mathrm{CDCl}_{3}\right): \delta \mathrm{H}$ 8.97(s, 2H), 8.33(d, 2H), 7.85-7.82(d, 2H), 7.567.43(m, 2H), 7.22-7.13(m, 6H), 7.01-7.04(d, 2H), 6.58(d, 2H), 3.9(s, 6H); ${ }^{13} \mathrm{C}$ NMR: $\delta \mathrm{C}$ 159.7, 156.0, 149.6, 132.2, 131.7, 128.2, 126.8, 126.7, 126.3, 125.7, 121.1, 118.5, 111.2, 55.6; $\mathrm{C}_{28} \mathrm{H}_{24} \mathrm{~N}_{2} \mathrm{O}_{2} \mathrm{~S}_{2}$ found (Calc.) C 69.0(69.4), H 4.3(4.9), N 6.2(5.8). 


\section{$N, N^{\prime}(1,1$ '-Dithiobis phenylene) bis p-anisalimine (6)}

Colour: yellow; ${ }^{1} \mathrm{H}$ NMR $\left(\mathrm{CDCl}_{3}\right)$ : $\delta \mathrm{H}$ 8.42(s, 2H), 8.05(d, 4H), $7.65(\mathrm{~d}, 2 \mathrm{H}), 7.16-6.99$ (m, $10 \mathrm{H}), 3.89$ (s, 6H); ${ }^{13} \mathrm{C}$ NMR: $\delta \mathrm{C} 162.6,159.3,149.2,132.0,131.0,129.2,126.8,126.5$, 125.8, 117.2, 114.4, 114.3, 55; $\mathrm{C}_{28} \mathrm{H}_{24} \mathrm{~N}_{2} \mathrm{O}_{2} \mathrm{~S}_{2}$ found (Calc.) C 68.8(69.4), H 4.6(4.9), N 6.2 (5.8), S 13.8(13.2).

\section{$N, N^{\prime}$ (1,1'-Dithiobis phenylene) bis pyridylimine (7)}

Colour: yellow; ${ }^{1} \mathrm{H}$ NMR $\left(\mathrm{CDCl}_{3}\right): \delta \mathrm{H}$ 8.67-8.65(d, 2H), 8.60(s, 2H), 8.34-8.30(d, 2H), 7.827.75(t, 2H), 7.62-7.58(d, 2H), 7.36-7.31(t, 2H), 7.19-7.04(m, 6H); ${ }^{13} \mathrm{C}$ NMR: $\delta \mathrm{C} 160.4$, $154.5,149.7,147.8,136.8,132.7,127.9,127.1,126.0,125.5,122.2,117.3 ; \mathrm{C}_{24} \mathrm{H}_{18} \mathrm{~N}_{4} \mathrm{~S}_{2}$ found (Calc.) C 68.2(67.6), H 4.2(4.2), N 13.4(13.1), S 15.5(15.0).

$N, N^{\prime}(1,1$ '-Dithiobis phenylene) bis-cinnamaldehydimine $(8)$

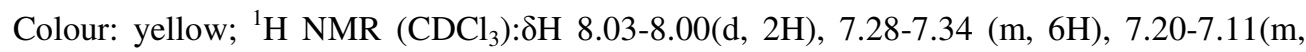
$8 \mathrm{H}), 6.87-6.94(\mathrm{~m}, 5 \mathrm{H}), 6.75-6.72(\mathrm{~m}, 2 \mathrm{H}), 6.44(\mathrm{dd}, 1 \mathrm{H}) ; \mathrm{C}_{30} \mathrm{H}_{22} \mathrm{~N}_{2} \mathrm{~S}_{2}$ found (Calc.) $\mathrm{C}$ 75.2(75.6), H 5.4(5.1), N 6.4(5.9).

\section{$N$-Phenyl-vanillaldimine (9)}

Colour: cream; ${ }^{1} \mathrm{H}$ NMR $\left(\mathrm{CDCl}_{3}\right): \delta \mathrm{H} 8.43(\mathrm{~s}, 1 \mathrm{H}) 7.52(\mathrm{~d}, 1 \mathrm{H}), 7.41-7.31(\mathrm{~m}, 3 \mathrm{H}), 7.21-$ 7.18(m, 3H), 6.96-6.87 (d, 1H) $3.38(\mathrm{~s}, 3 \mathrm{H}) ;{ }^{13} \mathrm{C}$ NMR: $\delta \mathrm{C} 161.4,153.2,151.4,149.2$, 130.4, 129.1, 126.6, 125.4, 122.1, 116.6, 111.6, 56.8; $\mathrm{C}_{14} \mathrm{H}_{13} \mathrm{NO}_{2}$ found (Calc.) C 73.8(74.0), H 5.7(5.7), N 6.2(6.2).

$N$-p-Tolylvanillaldimine (10)

Colour: yellow ${ }^{1} \mathrm{H}$ NMR $\left(\mathrm{CDCl}_{3}\right): \delta \mathrm{H} 8.43(\mathrm{~s}, 1 \mathrm{H}) 7.50(\mathrm{~s}, 1 \mathrm{H}), 7.32-7.29(\mathrm{~d}, 1 \mathrm{H}), 7.19-$ $7.10(\mathrm{~m}, 4 \mathrm{H}), 6.89-6.86(\mathrm{~d}, 1 \mathrm{H}), 3.83(\mathrm{~s}, 3 \mathrm{H}), 2.30(\mathrm{~s}, 3 \mathrm{H}) ;{ }^{13} \mathrm{C} \mathrm{NMR}: \delta \mathrm{C} 160.5,151.3,150.5$, 149.2, 135.8, 130.9, 129.2, 125.2, 122.0, 116.6, 111.6, 56.8, 21.8; $\mathrm{C}_{15} \mathrm{H}_{15} \mathrm{NO}_{2}$ found (Calc.) C 74.2(74.7), H 6.3(6.2), N 5.7(5.8).

\section{$\mathrm{N}$-o-Hydroxyphenyl (vanillaldimine) (11)}

Colour: brown; ${ }^{1} \mathrm{H}$ NMR (DMSO-d6): $\delta \mathrm{H} 8.82(\mathrm{~s}$,

$1 \mathrm{H}), 7.26(\mathrm{~d}, 2 \mathrm{H}), 7.08(\mathrm{~d}, 2 \mathrm{H}), 6.95-6.86(\mathrm{~m}, 3 \mathrm{H}), 6.34(\mathrm{~s}, 1 \mathrm{H}), 5.58(\mathrm{~s}, 1 \mathrm{H}), 3.81(\mathrm{~s}, 3 \mathrm{H})$ $\mathrm{C}_{14} \mathrm{H}_{13} \mathrm{NO}_{3}$ found (Calc.) C 68.5(69.1), H 5.0(5.4), N 6.0(5.76).

4-[(4-Hydroxy-3-methoxy benzylidine (amino) benzoic acid (12)

Colour: orange; ${ }^{1} \mathrm{H}$ NMR (DMSO-d6): $\left.\delta \mathrm{H} \mathrm{9.74(s,} \mathrm{1H),} \mathrm{7.66(d,} 1 \mathrm{H}\right), 7.41(\mathrm{~d}, 1 \mathrm{H}), 7.38(\mathrm{~s}$, $1 \mathrm{H}), 7.19(\mathrm{t}, 1 \mathrm{H}), 6.95(\mathrm{~d}, 1 \mathrm{H}), 6.71(\mathrm{~d}, 2 \mathrm{H}), 6.47(\mathrm{t}, 1 \mathrm{H}), 3.81(\mathrm{~s}, 3 \mathrm{H}) \mathrm{C}_{15} \mathrm{H}_{13} \mathrm{NO}_{4}$ found (Calc.) C 66.8(66.4), H 4.5(4.8), N 5.8(5.2).

\section{Results and Discussion}

In the past we have reported the synthesis of a number of Schiff bases using the classical method $^{11}$. On the other hand, an alternative method having green chemistry approach might lead to the formation of imines eliminating the generation of the hazardous substances. Herein, the synthesis of Schiff bases using mechanochemistry, which deals with the use of grinding to promote reactions between solid-solid or solid-liquid reactants were demonstrated. 
Reactions of diphenyldisulphide diamine with aldehydes or $p$-vanillin with amines were achieved by simply grinding together the starting materials in the molar ratio 1:2. Some of the reaction mixtures after grinding become viscous liquids or paste even where all reagents are solids. In some cases the melt phase stiffens within minutes to yield a sticky solid that hardens further on standing. The reactions were completed within 5-20 min with good yields comparable with classical methods. Reaction mixtures of vanillin were poured in ice after grinding. The product precipitated after 2 days on standing.

The synthesized products were characterized on the basis of their IR, NMR, elemental analysis and melting points (Table 1) and were compared with the data of products obtained from classical method ${ }^{11-13}$.

The Schiff bases (1-12) showed a strong signal in the region $1620 \mathrm{~cm}^{-1}$, assigned to the azomethine group. The NMR data $\left({ }^{1} \mathrm{H}\right.$ and $\left.{ }^{13} \mathrm{C}\right)$ are reported in the experimental section. The comparison of the ${ }^{1} \mathrm{H}$ NMR spectrum of 7 (Figure 1) obtained from both green and classical methods ${ }^{14}$ was comparable, hence confirming that the same product was formed. To the best of our knowledge the synthesis of $\mathbf{8}$ has not been reported. The ${ }^{1} \mathrm{H}$ NMR of $\mathbf{8}$ (Figure 2) showed the azomethine proton as a doublet at $\delta 8.02$ and the aromatic protons at $6.87-7.35 \mathrm{ppm}$. One of the olefinic protons appeared at $\delta 6.44$ as doublet of doublet while the other olefin proton was merged with the aromatic protons. The peaks at 7.8 and $6.2 \mathrm{ppm}$ indicated the formation of another isomer of the Schiff base 8. This may be due to resonance of the conjugated system.

Table 1. Solvent free synthesis of imines derivatives.

\begin{tabular}{|c|c|c|c|}
\hline & & Yield \% & M.P, ${ }^{0} \mathrm{C}$ \\
\hline Entry & Product & $\begin{array}{c}\text { Green } \\
\text { (Classical) }\end{array}$ & $\begin{array}{c}\text { Green } \\
\text { (Classical) }\end{array}$ \\
\hline 1 & $\begin{array}{l}N, N^{\prime}\left(1,1^{\prime} \text {-Dithiobisphenylene) bis }\right. \\
\text { benzyldeimine }\end{array}$ & $93(85)$ & $138(140)$ \\
\hline 2 & $\begin{array}{l}N, N^{\prime}(1,1 \text { '-Dithiobisphenylene) bis-2- } \\
\text { chlorobenzyldeimine }\end{array}$ & $97(98)$ & $172(173)$ \\
\hline 3 & $\begin{array}{l}N, \mathrm{~N}^{\prime}(1,1 \text { '-Dithiobisphenylene) bis-2- } \\
\text { nitrobenzyldeimine }\end{array}$ & $94(61)$ & $164(168)$ \\
\hline 4 & $\begin{array}{l}N, N^{\prime}\left(1,1^{\prime} \text {-Dithiobisphenylene }\right) \\
\text { bisfurfurylimine }\end{array}$ & $94(83)$ & $106(108)$ \\
\hline 5 & $\begin{array}{l}N, \mathrm{~N}^{\prime}(1,1 \text { '-Dithiobisphenylene) bis-o- } \\
\text { anisalimine }\end{array}$ & 94 & 163 \\
\hline 6 & $\begin{array}{l}N, N^{\prime}\left(1,1^{\prime} \text {-Dithiobis phenylene) bis p- }\right. \\
\text { anisalimine }\end{array}$ & 96 & $169(172)$ \\
\hline 7 & $\begin{array}{l}N, N^{\prime}\left(1,1^{\prime} \text {-Dithiobis phenylene) bis }\right. \\
\text { pyridylimine }\end{array}$ & $92(80)$ & $136(140)$ \\
\hline 8 & $\begin{array}{l}N, N^{\prime}\left(1,1^{\prime} \text {-dithiobisphenylene }\right) \text { bis- } \\
\text { cinnamaldehydimine }\end{array}$ & 98 & 138 \\
\hline 9 & $N$-Phenyl-vanillaldimine & $90(89)$ & $154(155)$ \\
\hline 10 & $N$-p-Tolylvanillaldimine & 92 & $154(158)$ \\
\hline 11 & $\mathrm{~N}$-o-Hydroxyphenyl (vanillaldimine) & 91 & $154(158)$ \\
\hline 12 & $\begin{array}{l}\text { 4-[(4-Hydroxy-3-methoxy benzylidine } \\
\text { (amino) benzoic acid }\end{array}$ & 89 & 164 \\
\hline
\end{tabular}


<smiles>Nc1ccccc1SSc1ccccc1N</smiles><smiles>[R]C=Nc1ccccc1SSc1ccccc1N=C[R]</smiles><smiles>COc1cc(C=O)ccc1O</smiles><smiles>[R]N=Cc1ccc(O)c(OC)c1</smiles>

Scheme 1. Synthesis route to imines.
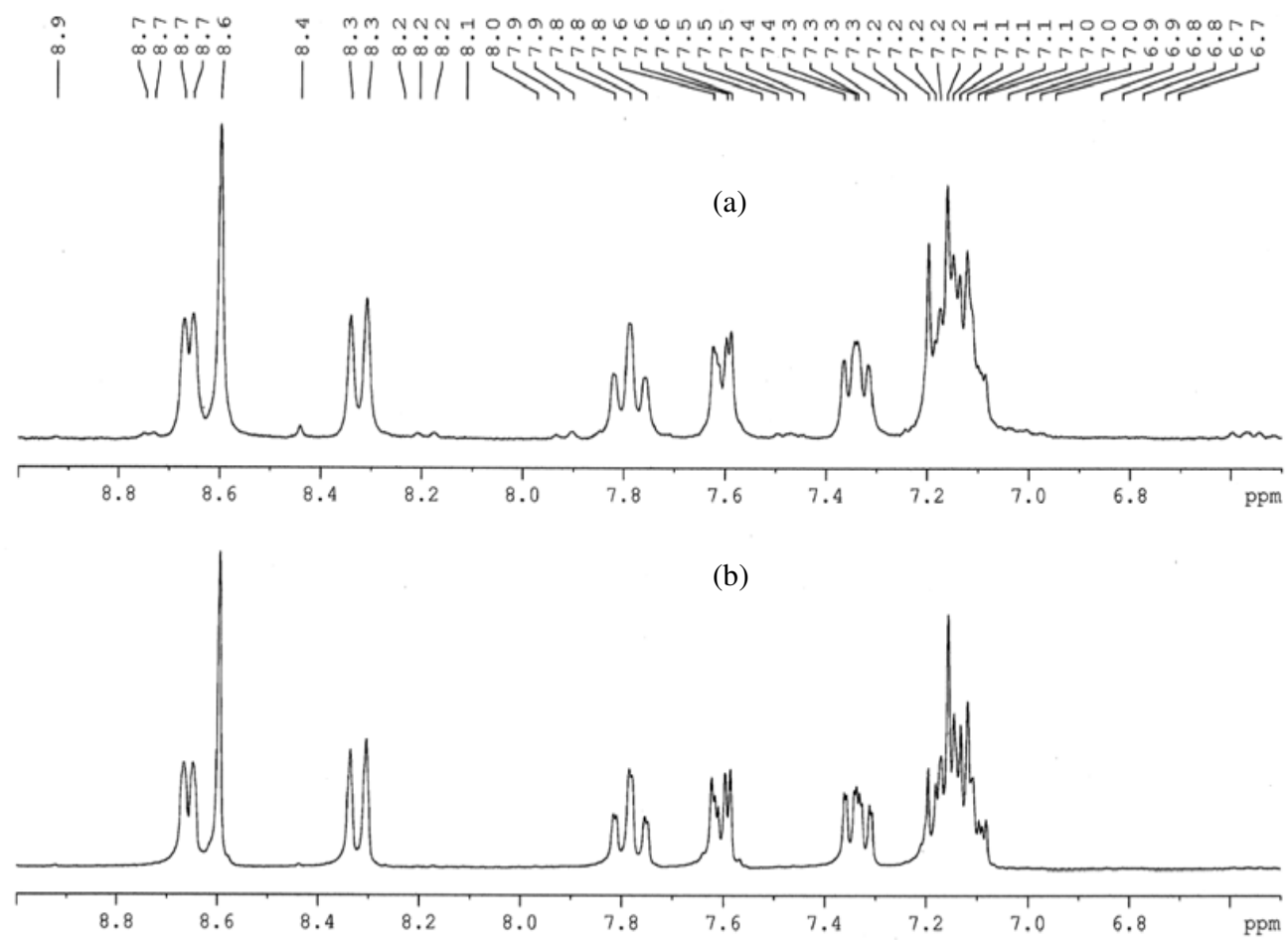

Figure 1. ${ }^{1} \mathrm{H}$ NMR spectra of 7 obtained from (a) green chemistry and (b) the classical method. 


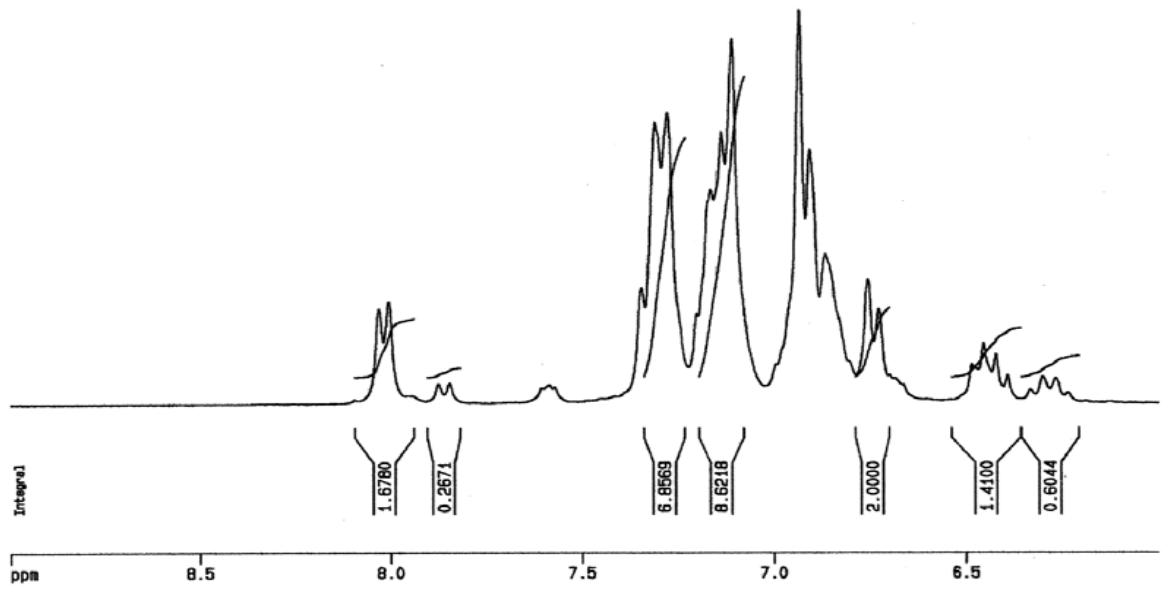

Figure 2. ${ }^{1} \mathrm{H}$ NMR spectra of compound 8 .

\section{References}

1. Sheldon R A, Green Chem., 2005, 7, 267- 278.

2. Hou X L, Wu J, Fan R H, Ding C H, Luo Z B and Dai L X, Synlett., 2006, 181-193.

3. Silveira C C, Vieira A S and Braga A, Tetrahedron, 2005, 61, 9312-9318.

4. Leitner W, Applied Organometallic Chemistry, 2000, 4(12), 209-814.

5. Yadav J S, Subba Reddy V, Shubashree S, Sadashiv K and Krishna Rao D, J Mol Catal A: Chemical., 2007, 272(1-2) 128-131.

6. Van den Ancker T R, Cave G W V and Raston C L, Green Chem., 2006, 8, 50-53.

7. Roberts BA, Cave G W V, Raston C L and Scott J L, Green Chem., 2001, 3, 280284; Raston C L and Scott J L, Green Chem., 2000, 4, 49-52.

8. Correa W H, Papadopoulos S, Radnidge P, Roberts B A and Scott J L, Green Chem., 2002, 4, 245-251; Correa W H and Scott J L, Green Chem., 2001, 3, 296-301.

9. Li C and Wei C, Chem Commun., 2002, 268-269; Ballini R, Fiorini D, Victoria Gill M and Palmieri A, Green Chem., 2003, 5, 475-476; Gopalakrishnan M, Sureshkumar P, Kanagarajan V, Thanusu J and Govindaraju R, ARKIVOK, 2006, 13, 130-141.

10. Bergman Y, Perlmutter P and Thienthong N, Green Chem., 2004, 6, 539-540; Wang Z X and Qin H L, Green Chem., 2004, 6, 86-92; Jain S L, Singhal S and Sain B Green Chem., 2007, 9, 740-741.

11. Bhowon M G, Jhaumeer-Laulloo S, Dowlut M, Curpen S and Jumnoodoo V Transition Met Chem., 2005, 30, 35-39.

12. Chanda, S, Vaghasiya K, Nair R and Baluja M S, J Serb Chem Soc. 2004, 69(12), 991-998.

13. Branko K and Gordana P, Acta Cryst., 1995, C51, 1875-1878.

14. Livingstone S E and Nolan J D, Aust J Chem., 1973, 26, 961-970. 


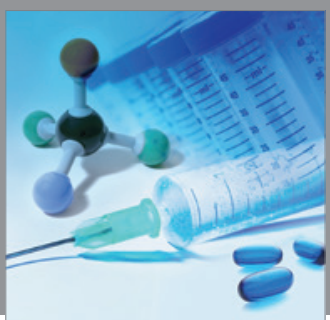

International Journal of

Medicinal Chemistry

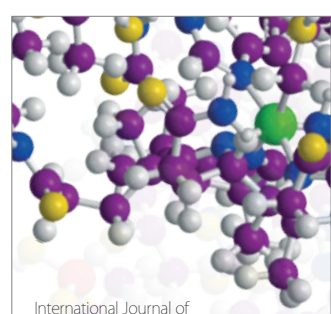

Carbohydrate Chemistry

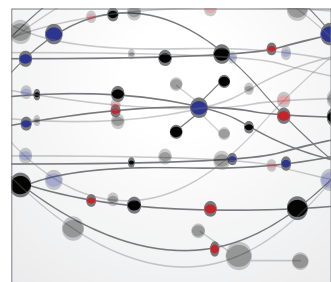

The Scientific World Journal
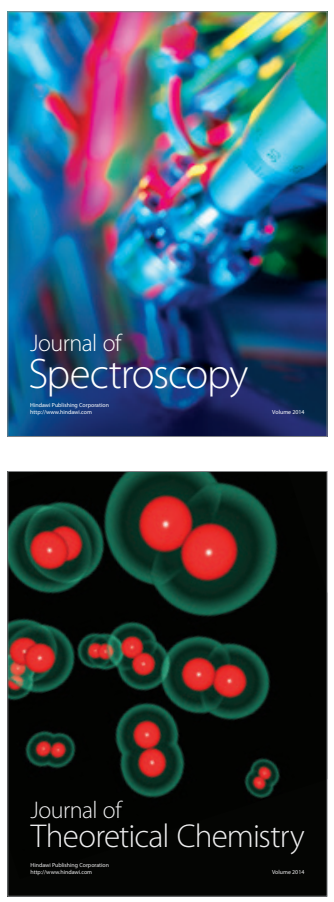
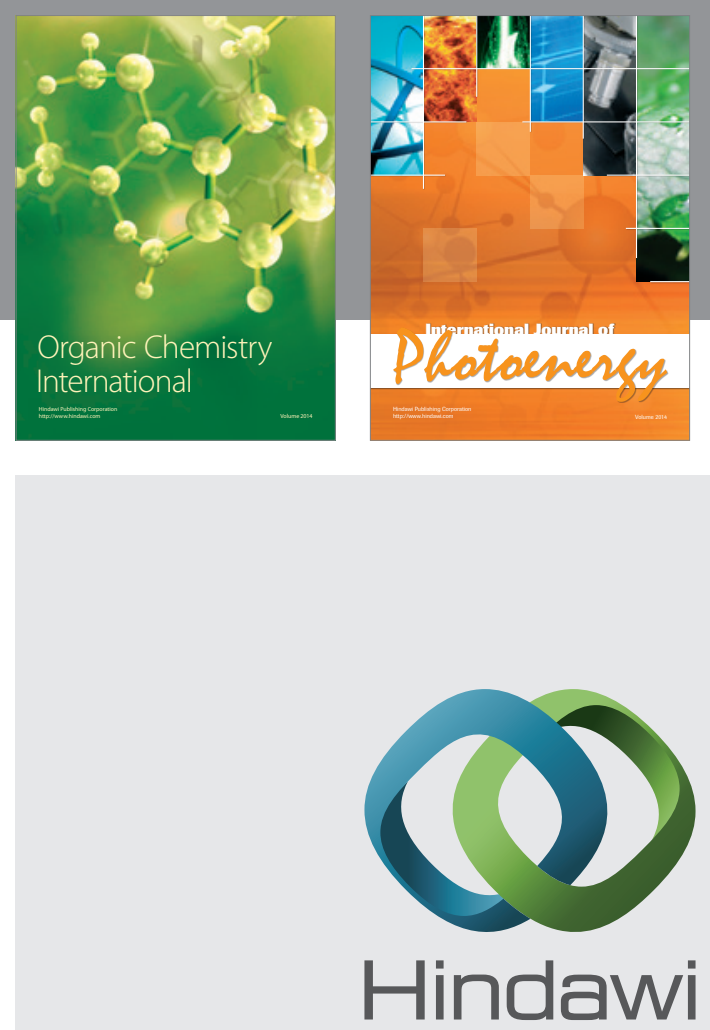

Submit your manuscripts at

http://www.hindawi.com
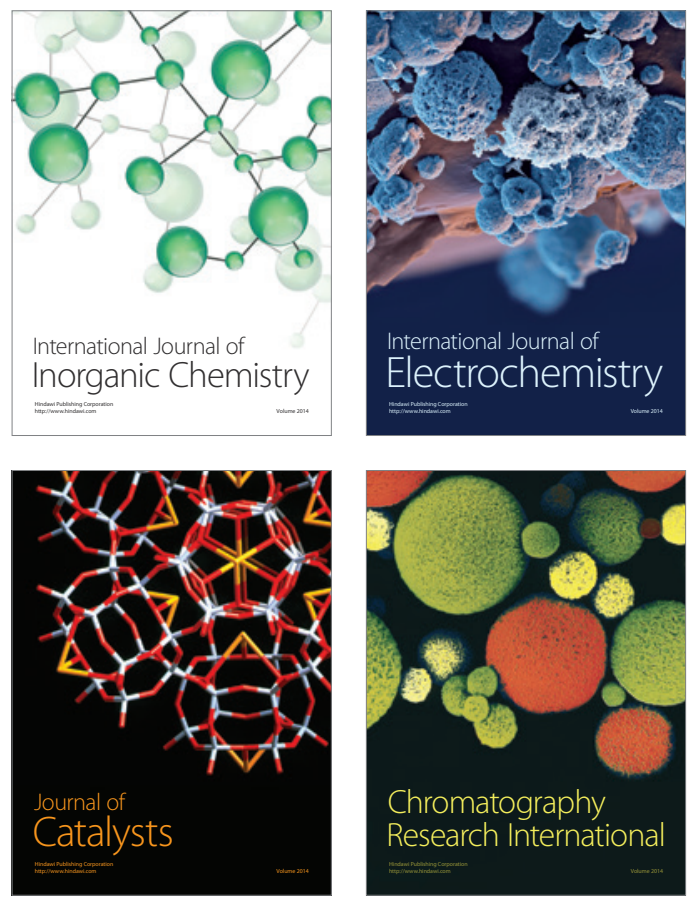
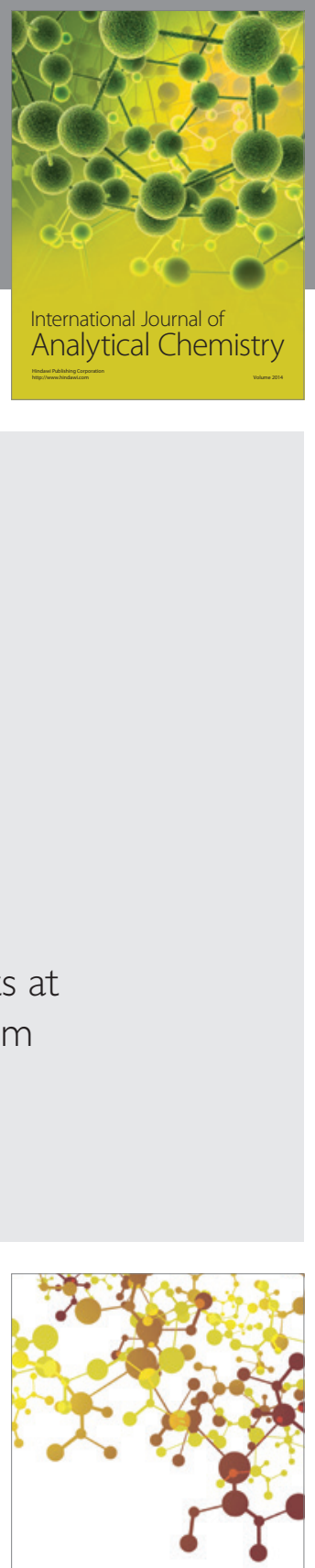

Journal of

Applied Chemistry
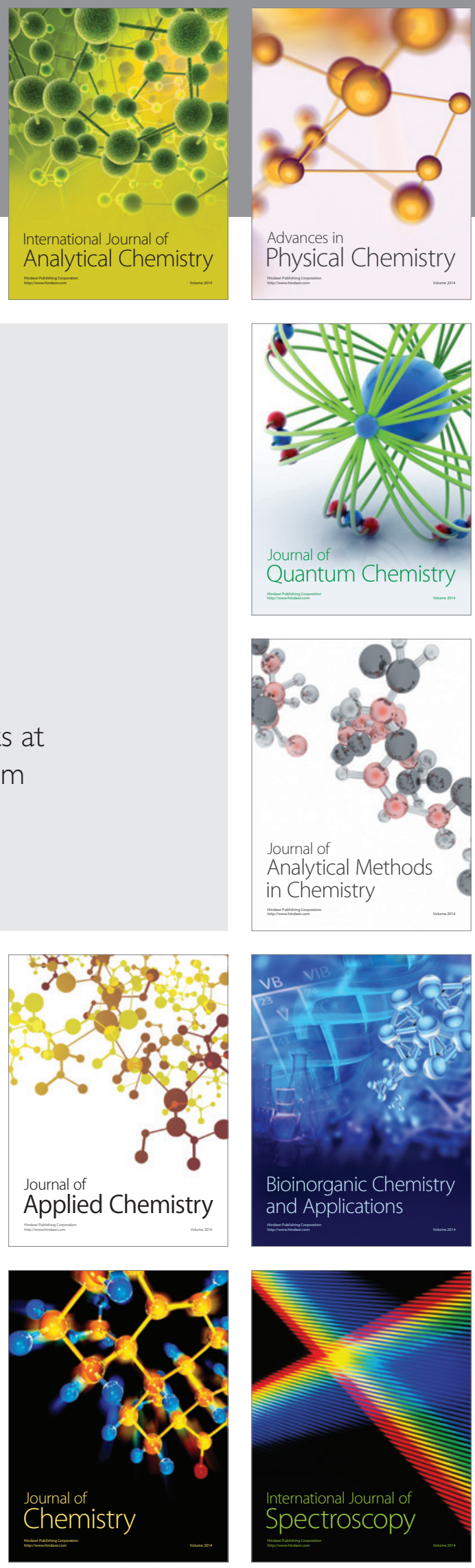